\title{
Design and application of intelligent monitoring terminal for boiler operating state
}

\author{
Shun Ma ${ }^{\text {a }}$, Hui Zhao, and Hong Wang \\ Hangzhou Special Equipment Inspection and Research Institute, 310051, Hangzhou, China \\ a mashunhappy@126.com
}

Keywords: industrial boiler; intelligent monitoring; terminal; embedded system

Abstract: In this paper, an intelligent monitoring terminal for boiler operating state was studied based on embedded technology, wireless communication technology, and highly integrated design technology. The architecture of intelligent monitoring system for industrial boiler was proposed firstly, and then the design of the hardware and software of the terminal was introduced. A STM32F103 ARM microprocessor, a SIM900A wireless module, a 7 inch serial touch screen, and $\mu \mathrm{C} / \mathrm{OS}-\mathrm{II}$ embedded operating system were used as the core of software and hardware of the terminal. Many functions such as data acquisition for mixed signal, GPRS wireless transparent transmission, field human-computer interaction, serial communication, online energy efficiency calculation, remote configuration management, were realized for all types of industrial boilers. Finally, the pilot application of the designed monitoring terminal was carried out. The application results show that the designed terminal can effectively realize the real-time monitoring and diagnosis for the energy saving and safety status of the industrial boiler. It is stable and reliable, and has good market prospects.

\section{Introduction}

Boiler is a mechanical heat exchanger that uses the heat of fuel or the waste heat in industrial production to heat the working medium to a certain temperature and pressure. However, there are many insufficiencies in the industrial boilers produced in China at present, such as low degree of automation, high energy consumption, low efficiency, serious pollution and accident prone [1]. In view of the existing regulatory means, it is difficult to concentrate on the centralized supervision of industrial boiler because of its small capacity, large quantity and scattered distribution [2]. The basic information and safety and energy saving dynamic regulatory data of all industrial boilers in the region is difficult to get, which will bring difficulties to the more comprehensive safety monitoring and energy consumption monitoring.

The intelligent monitoring system for boiler operating state based on the Internet of things technology is an important technical scheme to solve the above problems [3-5]. The real-time state parameters of the industrial boilers can be collected, coded, and transmitted to the remote management platform through the installation of sensors or the use of existing sensors of the boiler. Further, the safety and energy efficiency indicators of the monitored boilers can be analyzed, calculated and evaluated, and the operation situation of the monitored boilers can be released dynamically in real time on the Internet.

The front-end data acquisition and transmission device applied to the industrial field in the intelligent monitoring system of boiler status should be suitable for industrial boilers of various types and brands and various environment. However, in most of the existing studies, PLC or integrated data acquisition module combined with GPRS DUT device and commercial configuration software was used to achieve data acquisition and communication [6-7]. Such scheme 
has the advantages of fast system integration and short development cycle. But at the same time, there are many shortcomings, such as high production cost, poor compatibility and scalability, limited network transmission and poor on-site interaction. Therefore, it is difficult to promote the marketing of this kind of scheme.

In this paper, a general and intelligent monitoring terminal for boiler operating state, which is suitable for all kinds of furnaces and brands, is designed and applied by advanced technologies such as embedded control and wireless communication. The designed terminal can collect the operating parameters of the boiler in real time, monitor the operation and energy efficiency of each boiler in the area in a cost-effective manner, and evaluate the state and efficiency of the boiler in time. It provides a solid technical support for the centralized management and optimization of industrial boilers.

\section{Structure of intelligent monitoring system for industrial boiler}

The intelligent monitoring system for boiler operating state is a kind of Internet information system, in which the monitored equipment will increase increasingly, the application of the platform is complex and the data transmission needs to be stable and reliable. Thus, the Internet system architecture based on pre - data services and B/S network services should be used. The proposed overall architecture of the intelligent monitoring system is shown in Fig. 1. The system is composed of signal acquisition layer, data transmission layer, center management layer and user application layer.

As shown in Fig. 1, in this system, the monitored objects include all types of industrial boilers. The signal acquisition layer is composed of sensing elements and intelligent monitoring terminal. The sensing elements include all kinds of sensors and electrical components, such as temperature sensors, pressure sensors, flow sensors, oxygen content sensors, contactors, relays, etc., which are used to collect all kinds of physical signals of boiler. And the sensor can be an external sensor or an existing sensor in boiler according to the actual situation of the monitored object. The intelligent monitoring terminal for boiler operating state which is applied to the industrial field is the core of the whole monitoring system. It can monitor various physical parameters and states of industrial boilers by real-time sampling of the sensors, and automatically calculate the indirect monitoring parameters, such as the efficiency and energy consumption of industrial boilers according to the direct monitoring parameters, analyze the operating state of the boiler in real time and send out the alarm information under the abnormal condition of the boiler. Additionally, the terminal will show the real-time status of the boiler to the fireman through the man-machine interface and transfer the boiler monitoring parameters and information to the remote management platform through the data transmission network.

The data transmission layer of the system is GPRS/3G network and Internet network. The central management level is the remote monitoring and management platform for safety and energy saving of industrial boilers. And through the management, analysis and release of all monitoring data in the platform, the centralized supervision of all industrial boilers in the region can be realized. The hardware of the platform includes data acquisition server, database server, Web server, energy efficiency analysis and calculation server, short message machine, switchboard, firewall, etc.. And the software system of the platform, in which the B/S architecture is adopted, includes four parts: integrated sub-platform, government sub-platform, enterprise sub-platform and public sub-platform. Furthermore, the platform has seven functions, such as online monitoring, fault diagnosis and alarm, historical data query, multilevel authority management, information management, statistics and release of energy consumption data, and monitoring terminal management. 
The user application layer includes many browser-side users, such as government agencies, boiler inspection organizations, boiler use units, boiler manufacturing units, and boiler maintenance units, etc.. And it also supports mobile client users. The users can access the remote monitoring management platform through Internet or GPRS/3G network to obtain the real-time state data of the monitored boilers.

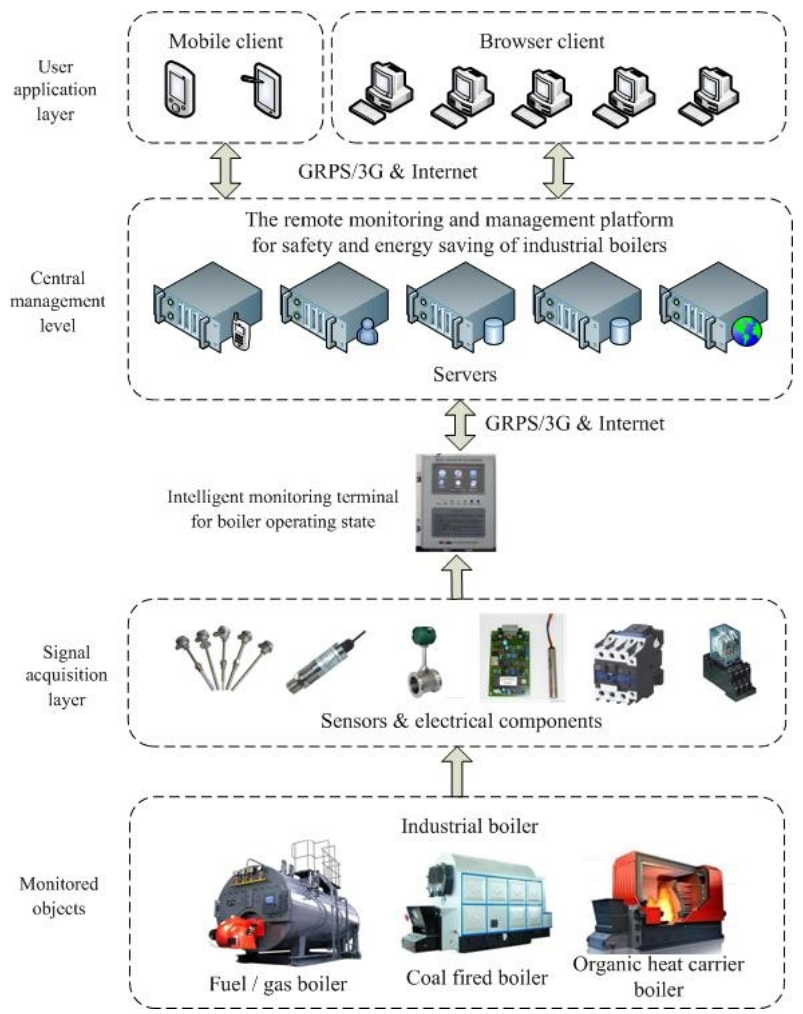

Fig. 1 Architecture of intelligent monitoring system for industrial boiler

\section{Hardware system design of monitoring terminal}

The designed intelligent monitoring terminal is composed of two parts: hardware and control software. And the hardware system consists of two parts, the circuit and the mechanical structure. The software and hardware design of the terminal is based on its functional characteristics, including analog signal and switch signal acquisition, serial communication, wireless communication, on-site human-machine interaction, data storage, real-time clock, online energy efficiency calculation, remote configuration and so on.

The overall structure of the designed hardware circuit is shown in Fig. 2. Host-slave computer structure is adopted in the circuit architecture. The circuit is composed of external sensor, CPU module, analog signal acquisition module, switch signal acquisition module, serial communication interface circuit, GPRS communication module, power module, display module and so on.

The external sensors that need to be installed on the monitored boiler include the sensors of temperature, pressure, flow rate, oxygen content and so on. According to the temperature range of each measurement point on the boiler, PT100 thermal resistance which has the characteristics of good stability, high precision and simple driving was chosen as the temperature sensor. And sensors with 4-20mA signal output were selected for pressure, flow, and oxygen content measurement.

The CPU module is responsible for data acquisition control, operation and communication processing. And the STM32F103 chip based on the ARM Cortex-M3 kernel was selected. The STM32 chip is designed for embedded applications with high performance, low cost and low power consumption. The working temperature range is $-40 \sim 85 \mathrm{C}$, and a large number of internal and 
external devices are integrated. Its performance and operation conditions meet the requirements of intelligent monitoring for industrial boilers.

The analog signal acquisition module includes the temperature measurement circuit and the 4-20mA signal sampling circuit. The MAX31865 chip with digital output which is combined with the four-wire PT100 was selected to realize the high precision measurement of each monitoring point. The measurement accuracy can reach $\pm 0.5^{\circ} \mathrm{C}$. The $\mathrm{I} / \mathrm{V}$ transform was adopted for the sampling of the current analog signals. A $140 \Omega$ precision resistor was used to convert the current signal to the voltage signal of $0.56 \mathrm{~V} \sim 2.8 \mathrm{~V}$, and the voltage signal was then transmitted to the ADC port of the CPU after the impedance transformation and filtering by the LM358 chip. The sampling resolution is $0.6835 \mathrm{mV}$, which meets the requirement of sampling precision. Besides, the serial differential input structure was adopted for the signal acquisition of the self-sensor of the boiler system, which can effectively avoid signal relay and prevent disturbance to the boiler system.

The function of the switch signal acquisition module is to isolate the extracted switch signal safely. The switch signals needed to be collected mainly are some switch and alarm information of the boiler itself, which will be slightly different according to the type of boiler. The switch signals were extracted from the relays, contactors and indicator lights in the boiler control cabinets. And the photoelectric isolator TLP521-4 was used to realize the acquisition of the dry contact switch signal. The relays combined with the photoelectric isolator PC817 were used to achieve the electrified contact switch signal acquisition.

The serial communication interface circuit adopts ADM2483 chip. The chip is combined with a protection circuit composed of transient suppression tube, common mode inductor, discharge tube, and self-recovery fuse to realize the RS485 communication with two channels, which has the characteristics of strong anti-interference ability and good electromagnetic compatibility.

The GPRS communication module is implemented by the SIM900A wireless module [8] with the industrial standard interface and the SMT encapsulation form, and the integrated AT instruction was used to achieve the interaction with the ARM microprocessor.

The power module that provides power to each module of system adopts an integrated switching power supply and voltage stabilizing chip LM2576 and LM1117-3.3 to realize the DC voltages of $24 \mathrm{~V}, 12 \mathrm{~V}, 5 \mathrm{~V}, 4.2 \mathrm{~V}$, and $3.3 \mathrm{~V}$.

The display module adopts an integrated 7 inch serial TFT LCD touch screen based on ARM kernel. The display unit acts as the host computer of the circuit system, and communicates with the slave computer (STM32F103) through the RS232 interface.

The mechanical structure of the monitoring terminal, which is composed of a shell and a cover, is made of aluminum metal. The main circuit board is fixed at the bottom of the shell, and the display unit, key, indicator lamp, etc. are fixed on the cover. The assembly structure and physical appearance of the monitoring terminal are shown in Fig. 3. 


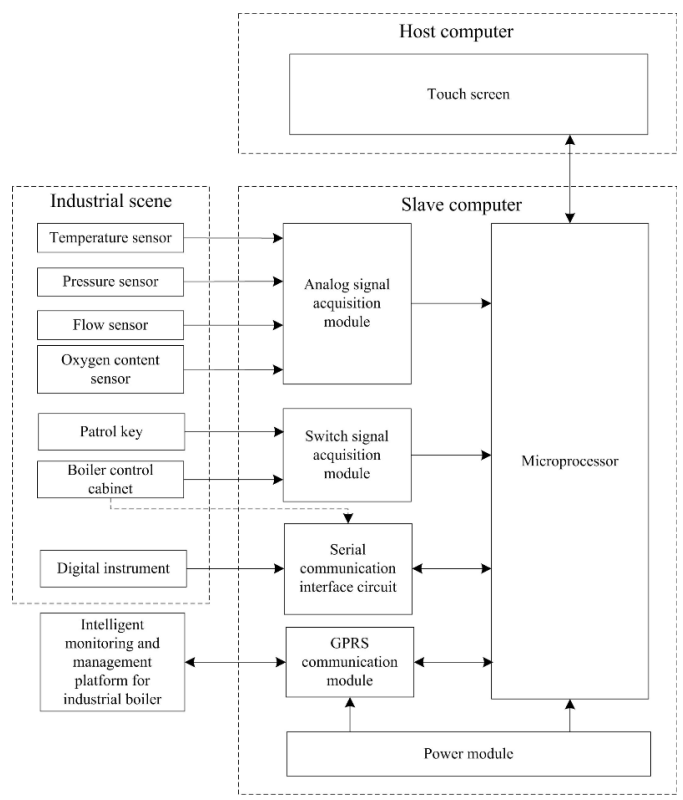

Fig. 2 Circuit system structure

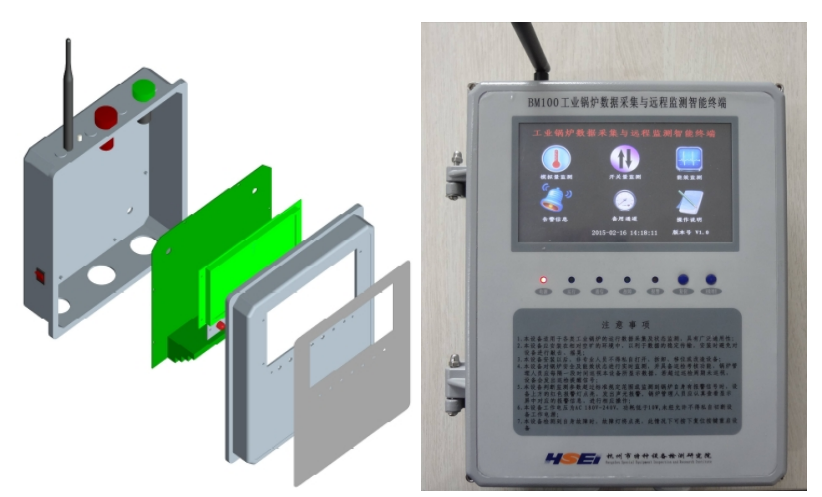

Fig.3 Terminal's assembly structure and photo

\section{Software system design of monitoring terminal}

The software system is based on the $\mu \mathrm{C} / \mathrm{OS}$-II kernel, which is the basis of task processing and process control. $\mu \mathrm{C} / \mathrm{OS}$-II has the characteristics of high efficiency, small space, good real-time performance and scalability. It is easy to transplant to a variety of microprocessors. The overall structure of the designed software system is shown in Fig. 4. The designed software is decomposed into a number of tasks, which run on the $\mu \mathrm{C} / \mathrm{OS}$-II operating system and complete all the work in collaboration. The system management task is responsible for the basic operations such as storage management, timing control and task switching. The analog signal processing task is mainly to complete the measurement of the analog signals such as temperature, pressure, oxygen content and so on. The switching signal processing task mainly monitors the switching signal state and performs the corresponding operation. The serial communication task is to deal with data communication, message construction, sending, receiving, analysis and so on. The GPRS communication task mainly completes remote data communication, and realizes the sending and receiving of data and control commands. The display management and man-machine interface task mainly complete the monitoring and processing of the touch screen, the indicator light and the buttons. The on-line energy efficiency calculation task is integrated with the mathematical models for energy efficiency calculation of each type of boiler. The mathematical model is established with reference to the related test rules in TSG G0003-2010 'energy efficiency test and evaluation rules for industrial boiler', in which energy efficiency parameters such as thermal efficiency, excess air factor, heat loss of exhaust smoke, heat loss of unburned gases, heat loss of unburned carbon, heat loss of radiation, physical heat loss of ash, etc. can be calculated according to the direct monitoring parameters, and the alarm information of the system can be evaluated according to the evaluation index of the boiler operation.

The interface of the developed software is shown in Fig. 5, which includes main interface, analog monitoring, switching value monitoring, energy efficiency monitoring, alarm information, spare channel and operation instructions. 


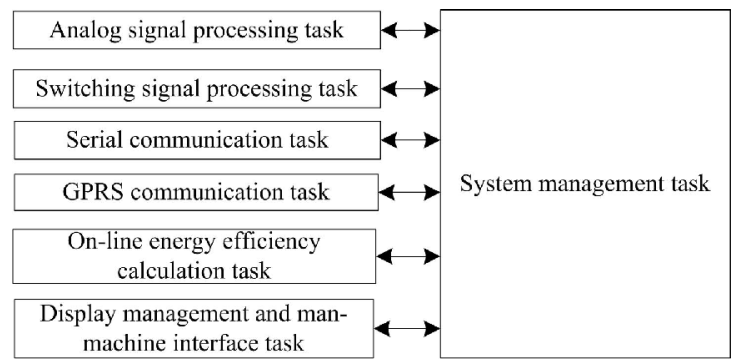

Fig. 4 Overall structure of software

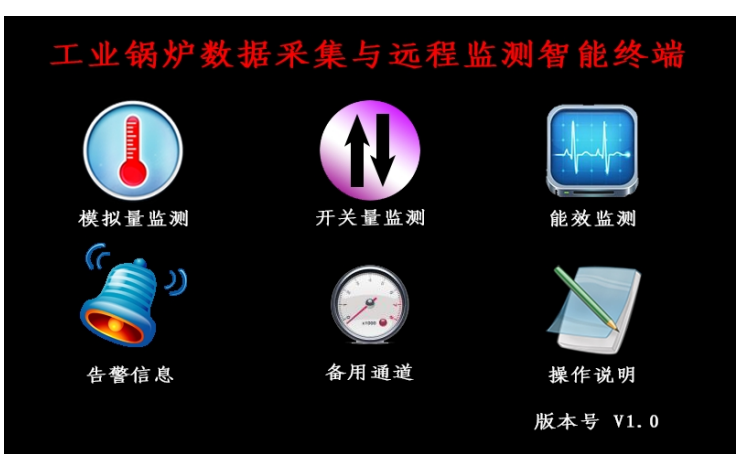

Fig. 5 Developed software interface

\section{Pilot application}

The pilot application of the designed monitoring terminal in the area of Hangzhou was carried out. The application of the pilot project in a boiler room of a hospital is shown in Fig. 6. The monitoring object is a 4 ton gas boiler with a model of WHS4-1.0-YQ. For this application, some additional sensors were installed on the monitored boiler. The monitoring points of steam temperature and pressure were arranged on the main steam pipes. The monitoring points of exhaust smoke temperature and exhaust smoke oxygen content were arranged on the exhaust pipe. The monitoring points of cold water temperature, hot water temperature and water hardness were arranged on the water supply pipes. The monitoring point of environmental temperature was arranged in the boiler room. The monitoring points of switch value were arranged in the boiler control cabinet, from which the switch signals were extracted, such as starting, ignition, alarm of high and low water level, overpressure alarm, comprehensive alarm and flameout fault. The prototype of the designed monitoring terminal was installed on the wall of the boiler room. All the monitoring terminals in the pilot project were successfully connected with 'the remote monitoring and service platform for energy saving and emission reduction of industrial boilers in Hangzhou' and have good operation effect. The effect of the monitoring terminal on the platform side is shown in Fig. 7.
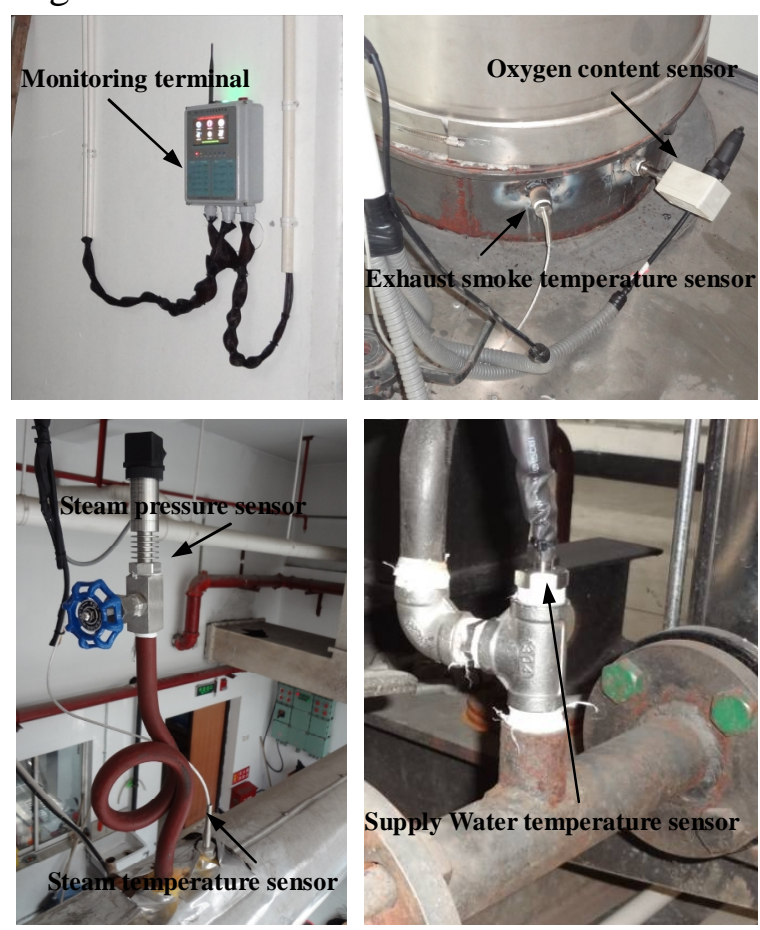

Fig. 6 Photos of pilot application

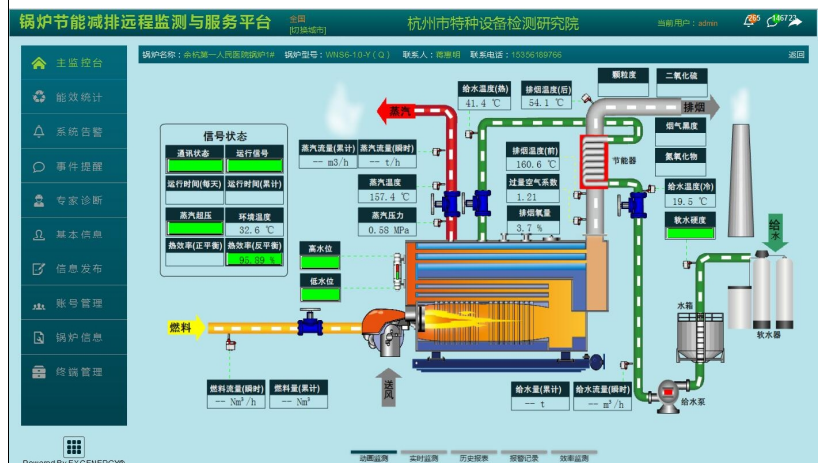

Fig. 7 System effect on the platform side 


\section{Conclusion}

An intelligent monitoring terminal for industrial boiler was designed by using embedded technology and wireless communication technology. The terminal uses the STM32F103 ARM microprocessor and the SIM900A wireless module as the core of the hardware, and uses the $\mu \mathrm{C} / \mathrm{OS}$-II embedded operation system as the core of the control software. It has functions of data acquisition for mixed signal, GPRS wireless transparent transmission, field human-computer touch interaction, serial communication, online energy efficiency calculation, and remote configuration management. The pilot application of the designed monitoring terminal was carried out. The test results show that the developed terminal can effectively realize the real-time monitoring and diagnosis for the energy saving and safety status of the industrial boiler, and has the characteristics of compact structure, high integration, low cost, good stability and reliability. And it has a good market prospect.

\section{Acknowledgements}

This work was financially supported by The Science and Technology Planning Project of General Administration of Quality Supervision, Inspection and Quarantine of the PRC (AQSIQ) (2014QK196), The Science and Technology Planning Project of Zhejiang Province (2014C33003), The Science and Technology Planning Project of Zhejiang Quality Supervision System (20160239), and The Science and Technology Development Planning Project of Hangzhou City (20131813A10).

\section{References}

[1] ZHAO Qin-xin, WANG Shan-wu. Developing Analysis for China's Industrial Boiler Future[J]. Industrial Boiler, 2007(1): 1-9.

[2] ZHAO Qin-xin, ZHOU Qu-lan. Solutions, Questions and Status of China's Industrial Boiler[J]. Industrial Boiler, 2010(1): 1-6.

[3] TONG Ying, GU Xinjian, JI Yangjian, et al. Online monitoring of industrial boiler based on IoT and cloud computing[J]. Computer Integrated Manufacturing Systems, 2016(1): 213-219.

[4] LI Fei-xiang, YANG Xiao-feng, WANG Hong, et al. Research and Development on Integrated System of Boiler Efficiency Test Based on the Internet of Things[J]. Industrial Boiler, 2013(4): 34-38.

[5] REN Qiang, ZHAO Hui. Application of Internet of Things to Boiler Supervision and Management[J]. Industrial Boiler, 2014(1): 50-54.

[6] DENG Shi-yang, LI Yao-ming. Remote Multi-point Monitoring System for Industrial Boiler Safety[J]. COMPUTER ENGINEERING, 2010, 36(17): 255-257.

[7] HUO Feng-cai, REN Wei-jian, KANG Chao-hai, et al. Design of Monitoring System for Industrial Boilers Based on KingView and PLC $[\mathrm{J}]$. Control and Instruments in Chemical Industry, 2013, 40(9):1103-1105.

[8]. HUO Tao, JIA Zhen-tang. Wireless communication module's design and implementation based on STM32 and SIM900A[J]. Electronic Design Engineering, 2014, 22(17): 106-110. 